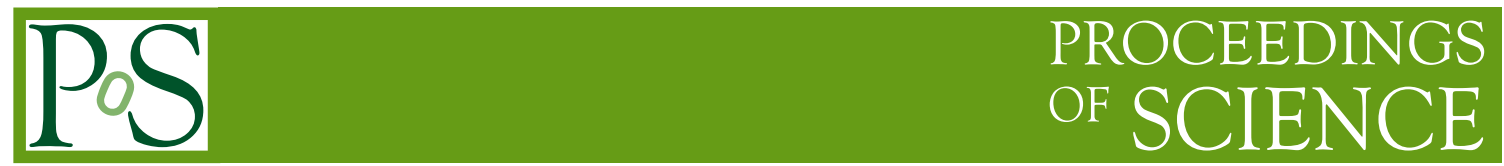

\title{
Search for QCD exotic states at CMS
}

\author{
Sergey Polikarpov on behalf of the CMS Collaboration ${ }^{a, *}$ \\ ${ }^{a}$ Lebedev Physical Institute of the Russian Academy of Sciences (LPI RAS) \\ Moscow, Russia \\ E-mail: sergey.polikarpov@cern.ch
}

The search for an exotic tetraquark state decaying into $\Upsilon(1 \mathrm{~S}) \mu^{+} \mu^{-}$performed at the CMS experiment is reported. An evidence for the $\mathrm{X}(3872)$ exotic state production in $\mathrm{PbPb}$ collisions is also presented, as well as the first observation of the $\mathrm{B}_{\mathrm{s}}^{0} \rightarrow \mathrm{X}(3872) \phi$ decay and the measurement of its branching fraction. The latter is found to be about two times smaller than $\mathcal{B}\left(\mathrm{B}^{+} \rightarrow \mathrm{X}(3872) \mathrm{K}^{+}\right)$, indicating the difference in the production dynamics of $\mathrm{X}(3872)$ in $\mathrm{B}_{\mathrm{s}}^{0}$ and $\mathrm{B}^{+}$decays.

40th International Conference on High Energy physics - ICHEP2020

July 28 - August 6, 2020

Prague, Czech Republic (virtual meeting)

\footnotetext{
${ }^{*}$ Speaker
} 
The CMS experiment [1] at the LHC is providing new important results in the Exotic Heavy Flavor physics sector. In this work, we report the search for a tetraquark state decaying into $\Upsilon(1 S) \mu^{+} \mu^{-}$(Section 1), an evidence for X(3872) production in nuclei collisions (Section 2), and the observation of the $\mathrm{B}_{\mathrm{s}}^{0} \rightarrow \mathrm{X}(3872) \phi$ decay (Section 3). Charge-conjugate states are implied throughout the text.

\section{Search for tetraquarks decaying into $\Upsilon(1 \mathrm{~S}) \mu^{+} \mu^{-}$}

A number of theoretical predictions exist for compact tetraquarks composed of two heavy quarks and two corresponding anti-quarks. In particular, the masses of bb $\overline{b b}$ tetraquarks are predicted to be around twice the $\eta_{\mathrm{b}}$ mass, slightly below twice the $\Upsilon(1 \mathrm{~S})$ mass. The CMS experiment performed a search [2] for such states in their decays into $\Upsilon(1 \mathrm{~S}) \mu^{+} \mu^{-}$, followed by the $\Upsilon(1 \mathrm{~S}) \rightarrow \mu^{+} \mu^{-}$decays, using the data collected in proton-proton (pp) collisions at $\sqrt{s}=13 \mathrm{TeV}$ in 2016 , corresponding to an integrated luminosity of $35.9 \mathrm{fb}^{-1}$. Four muons of high quality, having $p_{\mathrm{T}}>2.5 \mathrm{GeV}$, are selected and fit to a common vertex with $\chi^{2}$ probability in excess of $5 \%$. The mass of the first dimuon is required to be compatible with the known $\Upsilon(1 S)$ meson mass. If any opposite-sign dimuon is compatible with being a decay product of $\mathrm{J} / \psi$, the event is discarded.

The double- $\Upsilon(1 \mathrm{~S})$ production process is one of the background sources to the tetraquark search. Its contribution is estimated in data and its shape in the $\Upsilon(1 \mathrm{~S}) \mu^{+} \mu^{-}$mass distribution is evaluated using the simulated event samples. To improve the invariant mass resolution, the mass difference variable $M\left(\mu^{+} \mu^{-} \mu^{+} \mu^{-}\right)-M\left(\mu^{+} \mu^{-}\right)+m_{\Upsilon(1 \mathrm{~S})}$ is used, where the second term corresponds to the mass of the dimuon associated with $\Upsilon(1 \mathrm{~S}) \rightarrow \mu^{+} \mu^{-}$decay and $m_{\Upsilon(1 \mathrm{~S})}$ is the world-average mass of the $\Upsilon(1 \mathrm{~S})$ bottomonium state.

The observed distribution of $\Upsilon(1 \mathrm{~S}) \mu^{+} \mu^{-}$mass, presented in Fig. 1 (left), is fit with a sum of smooth background, $\Upsilon(1 \mathrm{~S}) \Upsilon(1 \mathrm{~S})$ contribution, and a signal component.
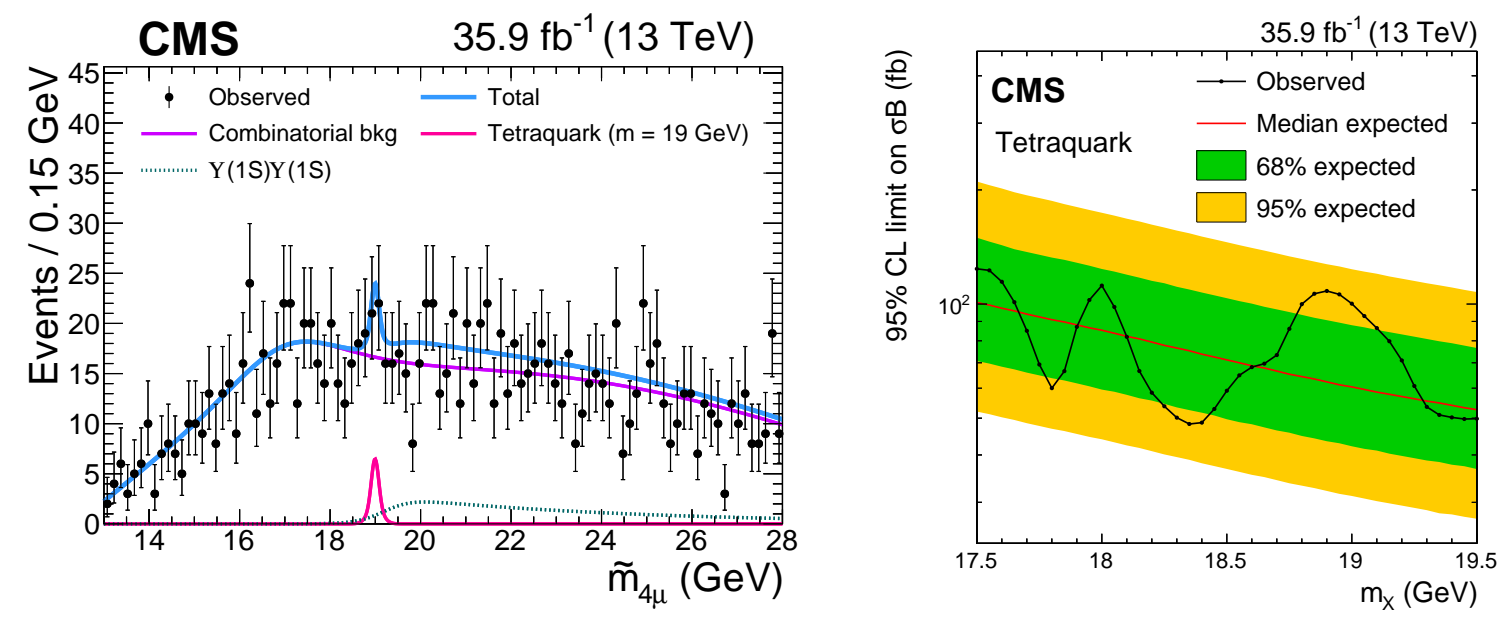

Figure 1: Left: the reconstructed $\Upsilon(1 \mathrm{~S}) \mu^{+} \mu^{-}$invariant mass distributions with the fit results overlaid [2]. Right: mass-dependent upper limit on the tetraquark production [2]. 
No significant signal is observed, and a mass-dependent upper limit is set on the production cross section multiplied by the branching fraction of the tetraquark decay into $\Upsilon(1 \mathrm{~S}) \mu^{+} \mu^{-}$and $\mathcal{B}\left(\Upsilon(1 \mathrm{~S}) \rightarrow \mu^{+} \mu^{-}\right)$, presented in Fig. 1 (right). The limit calculation relies on the reconstruction and identification efficiencies, estimated in simulations and verified in data. The limits are also set in a wider mass range on possible exotic particles decaying into $\Upsilon(1 \mathrm{~S}) \mu^{+} \mu^{-}$[2].

\section{Evidence for $\mathrm{X}(3872)$ production in relativistic heavy ion collisions}

Production of excited states of charmonium and bottomonium states is known to be suppressed in nucleus-nucleus collisions due to interactions with the hot medium. Moreover, higher excited states are suppressed more than lower excited states, for example, $\Upsilon(2 \mathrm{~S})$ is suppressed by a larger factor than $\Upsilon(1 \mathrm{~S})$ [3]. The X(3872) state, observed by the Belle experiment in 2003, is known to be inconsistent with a pure charmonium state due to its mass above double open charm threshold and small natural width. A number of interpretations exist, including molecule or tetraquark models of a superposition of those with a conventional charmonium state. Study of $\mathrm{X}(3872)$ production in nucleus-nucleus collisions and comparison to the $\psi(2 \mathrm{~S})$ production could bring additional information to improve the understanding of the $\mathrm{X}(3872)$ nature.

The CMS experiment performed such a study using $1.7 \mathrm{nb}^{-1}$ of $\mathrm{PbPb}$ collisions collected in 2018 at $\sqrt{s_{\mathrm{NN}}}=5.02 \mathrm{TeV}$. Both $\psi(2 \mathrm{~S})$ and $\mathrm{X}(3872)$ states are reconstructed through their decays into $\mathrm{J} / \psi \pi^{+} \pi^{-}$with a subsequent decay $\mathrm{J} / \psi \rightarrow \mu^{+} \mu^{-}$. The observed $\mathrm{J} / \psi \pi^{+} \pi^{-}$mass distribution is presented in Fig. 2, showing $\psi(2 \mathrm{~S})$ and $\mathrm{X}(3872)$ signals. The distribution is fit with a sum of a smooth background functions and two signal contributions. The statistical significance of the $\mathrm{X}(3872)$ signal is estimated to be about 4 standard deviations (s.d.), corresponding to the first evidence of $\mathrm{X}(3872)$ production in nuclei collisions [4]. The efficiency-corrected ratio of yields $N^{\text {corr }}(\mathrm{X}(3872)) / N^{\text {corr }}(\psi(2 \mathrm{~S}))$, after subtraction of non-prompt contribution, is measured to be $r=1.10 \pm 0.51$ (stat) \pm 0.53 (syst) [4]. This value is noticeably larger than that measured in pp collisions of $\sim 0.05-0.1$, however, due to large uncertainty in $r$, it is consistent with the pp value.

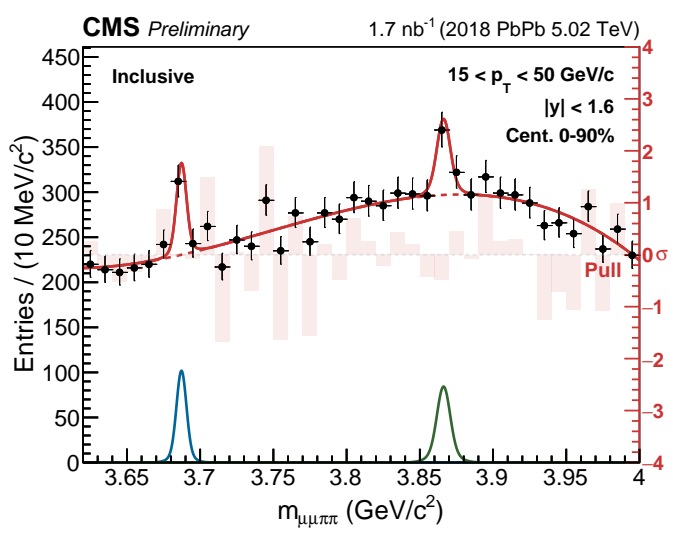

Figure 2: The $\mathrm{J} / \psi \pi^{+} \pi^{-}$invariant mass distribution observed in $\mathrm{PbPb}$ collisions. The left peak corresponds to the $\psi(2 \mathrm{~S})$ signal, the right peak - to the $\mathrm{X}(3872)$ [4]. 


\section{Observation of the $\mathrm{B}_{\mathrm{s}}^{0} \rightarrow \mathrm{X}(3872) \phi$ decay}

Studies of the $\mathrm{X}(3872)$ production in $\mathrm{b}$ hadron decays can provide important information for establishing the $\mathrm{X}(3872)$ internal structure. The exotic state has been previously observed in $\mathrm{B}^{+}$, $\mathrm{B}^{0}, \Lambda_{\mathrm{b}}^{0}$ decays, and in prompt production at hadron colliders.

The CMS experiment performed a search [5] for the $\mathrm{B}_{\mathrm{s}}^{0} \rightarrow \mathrm{X}(3872) \phi$ decay using pp collision data collected at $\sqrt{s}=13 \mathrm{TeV}$ in 2016-2018 and corresponding to an integrated luminosity of about $140 \mathrm{fb}^{-1}$. The signal decay is reconstructed using the $\mathrm{X} \rightarrow \mathrm{J} / \psi \pi^{+} \pi^{-} \rightarrow \mu^{+} \mu^{-} \pi^{+} \pi^{-}$and $\phi \rightarrow \mathrm{K}^{+} \mathrm{K}^{-}$ decays, and the normalization channel $\mathrm{B}_{\mathrm{s}}^{0} \rightarrow \psi(2 \mathrm{~S}) \phi \rightarrow \mathrm{J} / \psi \pi^{+} \pi^{-} \mathrm{K}^{+} \mathrm{K}^{-} \rightarrow \mu^{+} \mu^{-} \pi^{+} \pi^{-} \mathrm{K}^{+} \mathrm{K}^{-}$is chosen thanks to its identical final-state particles and very similar decay topology and kinematics.

The signal is extracted using a two-dimensional (2D) fit to $M\left(\mathrm{~K}^{+} \mathrm{K}^{-}\right)$and $M\left(\mathrm{~J} / \psi \pi^{+} \pi^{-}\right)$, after requiring $M\left(\mathrm{~J} / \psi \pi^{+} \pi^{-} \mathrm{K}^{+} \mathrm{K}^{-}\right)$to be in a narrow interval around the known $\mathrm{B}_{\mathrm{s}}^{0}$ meson mass. The measured $\mathrm{J} / 4 \pi^{+} \pi^{-}$invariant mass distribution is shown in Fig. 3 (left), overlaid with the projection of the $2 \mathrm{D}$ fit.

The 2D fit returns $299 \pm 39$ signal $\mathrm{X}(3872) \phi$ events and allows to estimate the statistical significance of above 6.5 s.d., corresponding to the first observation of the $\mathrm{B}_{\mathrm{s}}^{0} \rightarrow \mathrm{X}(3872) \phi$ decay. The remaining non- $\mathrm{B}_{\mathrm{s}}^{0}$ background is verified to be negligible using the ${ }_{\mathrm{s}} \mathcal{P}$ lot technique to subtract non-X(3872) and non- $\phi$ combinations $\mathrm{J} / \psi \pi^{+} \pi^{-} \mathrm{K}^{+} \mathrm{K}^{-}$and plotting the $\mathrm{J} / \psi \pi^{+} \pi^{-} \mathrm{K}^{+} \mathrm{K}^{-}$invariant mass distribution, as shown in Fig. 3 right. A similar signal extraction procedure is used in the normalization $\mathrm{B}_{\mathrm{s}}^{0} \rightarrow \psi(2 \mathrm{~S}) \phi$ channel to evaluate the yield $N\left(\mathrm{~B}_{\mathrm{s}}^{0} \rightarrow \psi(2 \mathrm{~S}) \phi\right)=15359 \pm 171$, which is then combined with the $\mathrm{B}_{\mathrm{s}}^{0} \rightarrow \mathrm{X}(3872) \phi$ yield and corrected for the efficiency ratio to obtain [5]

$$
\frac{\mathcal{B}\left(\mathrm{B}_{\mathrm{s}}^{0} \rightarrow \mathrm{X}(3872) \phi\right) \mathcal{B}\left(\mathrm{X}(3872) \rightarrow \mathrm{J} / \psi \pi^{+} \pi^{-}\right)}{\mathcal{B}\left(\mathrm{B}_{\mathrm{s}}^{0} \rightarrow \psi(2 \mathrm{~S}) \phi\right) \mathcal{B}\left(\psi(2 \mathrm{~S}) \rightarrow \mathrm{J} / \psi \pi^{+} \pi^{-}\right)}=(2.21 \pm 0.29 \text { (stat) } \pm 0.17 \text { (syst) }) \% .
$$

After multiplying this ratio by the known denominator branching fractions, one obtains $[5] \mathcal{B}\left(\mathrm{B}_{\mathrm{s}}^{0} \rightarrow\right.$ $\mathrm{X}(3872) \phi) \mathcal{B}\left(\mathrm{X}(3872) \rightarrow \mathrm{J} / \psi \pi^{+} \pi^{-}\right)=(4.14 \pm 0.54$ (stat) \pm 0.32 (syst) $\pm 0.46(\mathcal{B})) \times 10^{-6}$. This product is compared to the similar products of branching fractions for the $\mathrm{B}^{+} \rightarrow \mathrm{X}(3872) \mathrm{K}^{+}$and $\mathrm{B}^{0} \rightarrow \mathrm{X}(3872) \mathrm{K}^{(*) 0}$ decays in Fig. 4 (right). The $\mathrm{B}^{+} \rightarrow \mathrm{X}(3872) \mathrm{K}^{+}$decay evidently has about two times larger branching fraction with respect to the $\mathrm{B}^{0} \rightarrow \mathrm{X}(3872) \mathrm{K}^{(*) 0}$ and $\mathrm{B}_{\mathrm{s}}^{0} \rightarrow \mathrm{X}(3872) \phi$ decays,
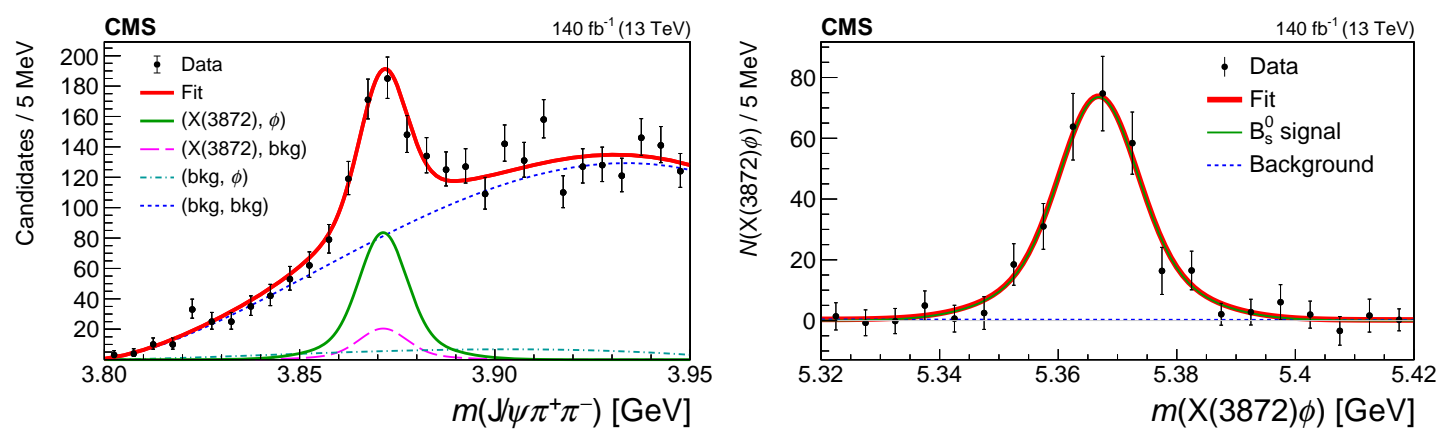

Figure 3: Left: $\mathrm{J} / 4 \pi^{+} \pi^{-}$invariant mass distribution in the selected $\mathrm{B}_{\mathrm{s}}^{0} \rightarrow \mathrm{X}(3872) \phi$ candidates in data [5]. Right: $\mathrm{X}(3872) \phi$ invariant mass distribution after subtraction of non- $\phi \mathrm{K}^{+} \mathrm{K}^{-}$combinations and non-X(3872) $\mathrm{J} / 4 \pi^{+} \pi^{-}$combinations [5]. 

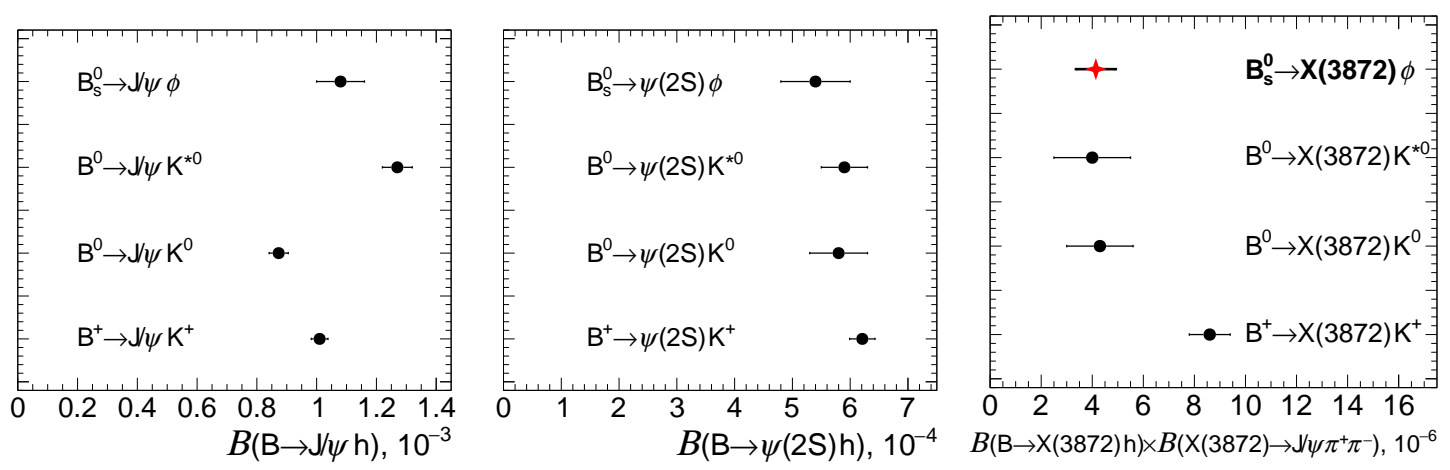

Figure 4: Comparison of branching fractions of B meson decays into a charmonium state (or $\mathrm{X}(3872)$ ) and a light meson [5].

in contrast to the similar decays of B mesons into $\mathrm{J} / \psi h$ and $\psi(2 \mathrm{~S}) h$, which have approximately equal branching fractions as shown in Fig. 4 left and center [5]. This shows that $\mathrm{X}(3872)$ production in $\mathrm{B}$ meson decays is different from that of traditional charmonium states.

\section{Summary}

In summary, the exotic tetraquark states decaying into $\Upsilon(1 \mathrm{~S}) \mu^{+} \mu^{-}$are searched for, and massdependent limits are set on their production cross section multiplied by the branching fractions. The first evidence for $\mathrm{X}(3872)$ state production in $\mathrm{PbPb}$ collisions and the first observation of $\mathrm{X}(3872)$ state production in $\mathrm{B}_{\mathrm{s}}^{0}$ meson decays are reported. The branching fraction of the $\mathrm{B}_{\mathrm{s}}^{0} \rightarrow \mathrm{X}(3872) \phi$ is found to be about two times smaller than that of the $\mathrm{B}^{+} \rightarrow \mathrm{X}(3872) \mathrm{K}^{+}$decay, indicating the difference in the production dynamics of $\mathrm{X}(3872)$ in $\mathrm{B}_{\mathrm{s}}^{0}$ and $\mathrm{B}^{+}$decays.

\section{Acknowledgments}

The author's work was supported by the programme of the presidium of the Russian Academy of Sciences (No. 3 "Physics of fundamental interactions and nuclear technology", 2018-2020) and by the Ministry of Science and Higher Education of the Russian Federation, Project "Fundamental properties of elementary particles and cosmology" No 0723-2020-0041. The author would like to thank the ICHEP 2020 conference organizers for the excellent program and opportunity to present these important results.

\section{References}

[1] The CMS Collaboration, The CMS experiment at the CERN LHC, JINST 3 (2008) S08004.

[2] The CMS Collaboration, Measurement of the $\Upsilon(1 S)$ pair production cross section and search for resonances decaying to $\Upsilon(1 S) \mu^{+} \mu^{-}$in proton-proton collisions at $\sqrt{s}=13 \mathrm{TeV}$, Phys. Lett. B 808 (2020) 135578. 
[3] The CMS Collaboration, Measurement of nuclear modification factors of $\Upsilon(1 \mathrm{~S}), \Upsilon(2 \mathrm{~S})$, and $\Upsilon(3 \mathrm{~S})$ mesons mesons in PbPb collisions at $\sqrt{s_{\mathrm{NN}}}=5.02 \mathrm{TeV}$, Phys. Lett. B 790 (2019) 270.

[4] The CMS Collaboration, Evidence for $\chi_{c 1}$ (3872) in PbPb collisions and studies of its prompt production at $\sqrt{s_{\mathrm{NN}}}=5.02 \mathrm{TeV}$, CMS-PAS-HIN-19-005.

[5] The CMS Collaboration, Observation of the $\mathrm{B}_{\mathrm{s}}^{0} \rightarrow \mathrm{X}(3872) \phi$ decay, arXiv:2005:04764, CMS-BPH-17-005, accepted by Phys.Rev.Lett. 